\title{
Soil moisture distribution around land mines and the effect on relative permittivity
}

\author{
Jan B. Rhebergen, Henk A. Lensen, Piet B.W. Schwering ${ }^{a}$, \\ Garciela Rodríguez Marín, Jan M.H. Hendrickx, ${ }^{b}$ \\ ${ }^{a}$ TNO Physics and Electronics Laboratory, The Hague, The Netherlands \\ ${ }^{b}$ New Mexico Tech, Socorro, New Mexico, USA
}

\begin{abstract}
Soil water content, relative permittivity, electrical conductivity, thermal conductivity and heat capacity, directly or indirectly affect the detection capabilities of sensors used for land mine detection. The most important of these is water content since it also influences the other properties. Therefore an experiment was set up where water was applied to a test area and the water content was monitored over time. Simultaneously, measurements with a ground penetrating radar (GPR) were carried out. Subsequently the measurements of both the water content reflectometers (WCR) and GPR were compared against the outcome of a soil water content model ${ }^{1}$ and a model relating soil water content with medium relative permittivity. ${ }^{2}$ We find that the introduction of water in a dry sand soil, increases the impedance contrast of the land mine with respect to its surrounding (i.e. stronger electromagnetic signatures) which may result in better detection. Alternative effects also seem play a role in finding and identifying features of potential targets.
\end{abstract}

Keywords: Mine detection, soil water distribution, modelling, anti tank mine, anti personnel mine, test facility, relative permittivity, dielectric constant, ground penetrating radar, GPR

\section{INTRODUCTION}

One of the candidate sensors for land mine detection is the Ground Penetrating Radar (GPR). Although GPR can be used as a separate mine detector e.g. in a tool box approach in addition to other mine detection equipment like metal detector and prodder, it is more likely to be included into a multi-sensor mine detection system. In such a system the processed GPR signals are 'fused' with the processed signals from other sensors. At some stage the contribution of each sensor to the detection of the mine and reduction of the false alarms is weighted in order to get the best detection results for a given situation (scenario). Obviously the performance of a multi-sensor system can be optimised amongst others by assigning improved weighting functions for each sensor. The weighting functions can be better determined when more knowledge of the detection process, the detection environment and the performance of the respective sensors is gained.

The detection performance of mine detection sensors is influenced by many factors, like type and depth of the land mine, soil texture and structure, soil water content and prevailing and historic weather conditions. The most important parameter that influences the performance of a GPR is the soil water content since this directly influences the electromagnetic parameters to which a GPR is sensitive namely the relative permittivity and electrical conductivity of the embedding soil environment. Through proper modelling using known quantities like bulk soil density and texture, as well as (historic) weather information like precipitation and temperature, the spatial moisture distribution around a buried object of certain dimensions like a land mine can be predicted.

The spatial moisture distribution derived from the model will be highly correlated to the permittivity and conductivity profile around the land mine. Thus the complex and frequency dependent electromagnetic impedance contrast of the land mine and its embedding can be directly influenced by changing the moisture conditions of the surrounding environment. In fact the attenuation of EM waves and the change in medium permittivity can to a certain degree be predicted. While increased attenuation will negatively influence detection

Send correspondence to: J.B. Rhebergen, TNO-FEL, P.O. Box 96864, NL-2509 JG The Hague, The Netherlands, Rhebergen@fel.tno.nl, phone: +31 703740772

Detection and Remediation Technologies for Mines and Minelike Targets VII, J. Thomas Broach, 
performance, because the incident and scattering signals will become weaker, the change in medium permittivity will have such an effect on the impedance contrast of the buried mine and its surrounding, that this may improve detection probability. This makes it possible to construct environmental conditions by means of 'smart' watering (or drying) that are optimal for land mine detection by means of GPR.

The goal of this paper is to measure the moisture distribution around a buried land mine and determine the effect on the electromagnetic impedance contrast of the land mine with respect to the surrounding soil environment. Additionally we compare the measurements to the outcome of a soil water model ${ }^{1}$ and a model relating soil water content to relative permittivity ${ }^{2}$ of the surrounding soil medium.

For the purpose of this experiment the soil water content was modelled around an anti-tank mine (type NR26) buried at a depth of $30 \mathrm{~cm}$ below the surface in a sand soil. In order to compare the model predictions with experimental data, several non-metal anti-tank mines (type NR26) were buried at a depth of $30 \mathrm{~cm}$ in a sand soil (dune sand). Water content reflectometers (WCR) were installed near and just below and above one of the mines. In this way the soil water content and the effect of the mines on the distribution of the water content could be monitored during the course of the experiment. For both the model and experiment a given amount of water was added instantaneously to the initially dry soil and its effect was modelled and monitored. The relative permittivity of the embedding soil environment was estimated using Topps ${ }^{2}$ empirical relation between soil water content and relative permittivity. In order to compare the estimated relative permittivity with real data, GPR measurements were carried out during (and after) the infiltration process of the water. The GPR used here was an ERA SPR-Scan fitted with a $1 \mathrm{GHz}$ antenna unit. The described experiment was carried out at a GPR test site located near the TNO Physics and Electronics Laboratory (TNO-FEL) in The Netherlands.

The idea is that through knowledge of the local soil characteristics and local climatic conditions, as well as a priori knowledge of the type of land mines that one can expect to find, one can judge GPR performance and use scenario information to decide what equipment to use and when and how to deploy it, under given circumstances.

\section{ENVIRONMENTAL AND GPR RELATED MODELLING}

GPR performance is dependent on many factors, the most important of which probably is the characteristics of the embedding (ground/soil) environment. The ground environment in turn depends intimately on prevailing and historic weather conditions i.e. moisture content. This section will briefly address the environment related effects encountered in GPR measurements and their impact on results. First we will model how water is distributed around an anti tank mine. Next we look at which aspects of GPR performance are possibly affected by the introduction of water in its environment and the mechanisms involved. Finally we wil use a model ${ }^{2}$ to relate soil water content to the electromagnetic characteristics that influence GPR measurements.

\subsection{Simulation of soil water distribution around anti tank mine}

The modelling as described in this section enables one to relate given soil characteristics and climatological information to soil water content distribution around land mines.

Soil water distributions around land mines have been modelled with the HYDRUS-2D simulation package ${ }^{1}$ of the U.S. Salinity Laboratory at Riverside, California. HYDRUS-2D is a Microsoft Windows based modelling environment for simulating two-dimensional water, heat, and solute movement and root water uptake in variably saturated soil. The flow equations are solved numerically using a Galerkin-type linear finite element scheme. The software includes a mesh generator and graphical user interface.

For the land mine problem, we have followed earlier work by Das ${ }^{3}$ et al. and Hendrickx ${ }^{4}$ et al. and used a quasi three-dimensional region exhibiting radial symmetry about the vertical axis. Richards equation was solved to simulate unsaturated water flow in a soil cylinder with a radius of $2 \mathrm{~m}$ and depth of $2 \mathrm{~m}$. The anti tank mine (type NR26) was placed in the centre of the cylinder with its top at a depth of $0.30 \mathrm{~m}$. A finite element mesh was generated by the mesh generator provided with the HYDRUS-2D program. Observation points were located approximately one centimetre above and one centimetre below the mine about $1 \mathrm{~cm}$ away from its centre. Simulations were conducted for a sand soil. Since no hydraulic properties have been measured we used the hydraulic properties of the Van Genuchten model provided by the HYDRUS-2D model. ${ }^{1}$ 
The initial condition was set as a dry sand soil (4\% volumetric water content) throughout the profile. $80 \mathrm{~mm}$ of water infiltrating in the second hour after start of the simulations was applied as top boundary condition. A no-flux boundary condition was imposed on two sides of the flow domain. The simulations were conducted for a period of 48 hours. It was assumed that no significant evaporation occurred from the soil during this period, which is reasonable for winter conditions in The Netherlands.

Figure 1 presents the changes in soil water content at depths 10, 30, and $45 \mathrm{~cm}$ for the infiltration experiment. The water contents at 30 and $45 \mathrm{~cm}$ depths are those just above and just below the centre of the mine.

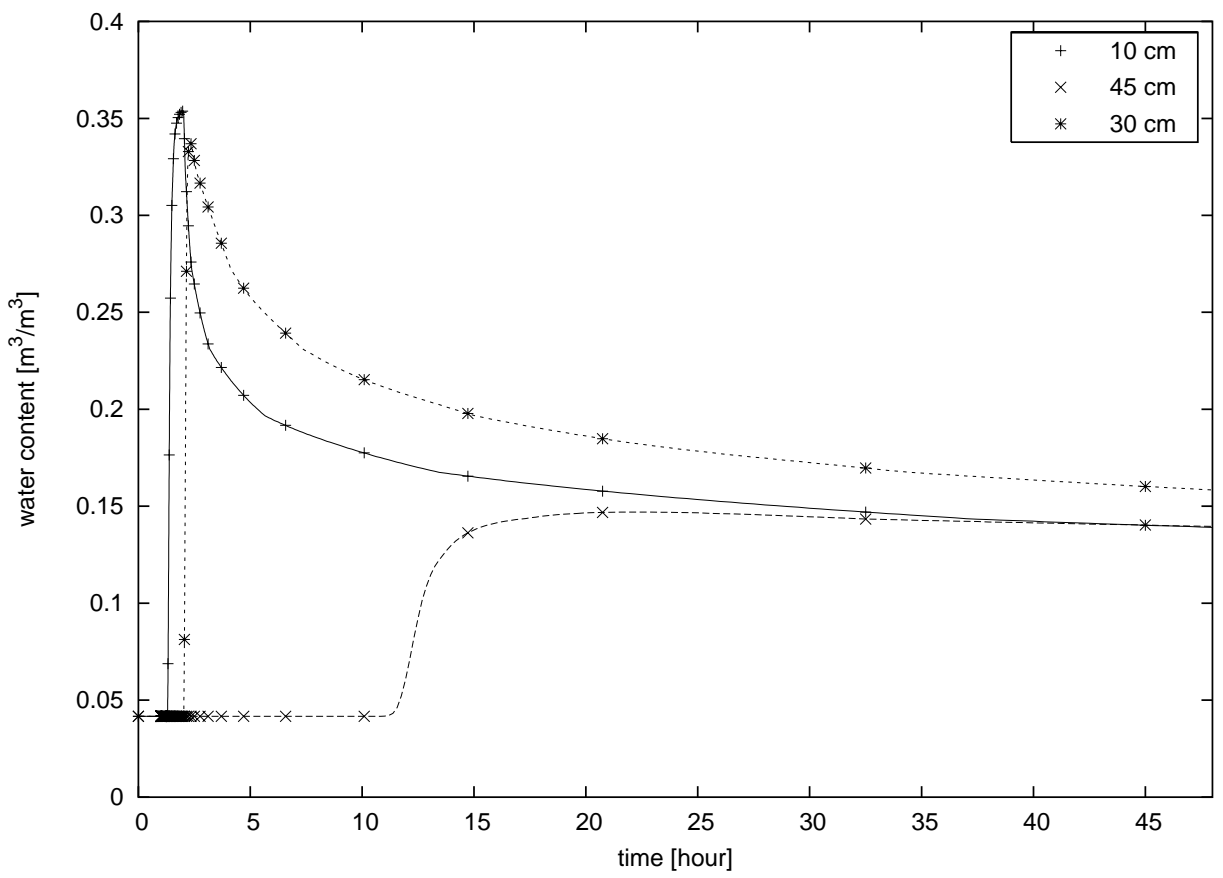

Figure 1: Modelled water content after $80 \mathrm{~mm}$ infiltration

Although water is applied only during one hour, soil water remains dynamic for the entire 48 hours of the simulation. After an application of $80 \mathrm{~mm}$ the soil water content just above the mine $(30 \mathrm{~cm}$ depth) increases almost immediately while below the mine $(45 \mathrm{~cm}$ depth) it takes about 10 hours after termination of water application at the soil surface for the water content to increase. All water contents stabilise at around 15 volume percent. This means that a layered soil profile due to water infiltration in a dry soil is a common phenomenon of field soils.

\subsection{Effects of moisture on GPR range \& resolution}

An important GPR parameter is its 'reach' or rather its range. This determines how deep and under which conditions a GPR can be used. The range of a GPR is mainly affected by the loss over the complete path of the signal between transmitter and receiver. There are three principal mechanisms contributing to this loss.

- material loss (embedding/soil)

- spreading loss (antenna)

- target reflection or scattering loss (objects)

The total path loss can be written as a sum of $\operatorname{losses}^{5}$ as show in equation 1 and elaborated in table 1

$$
L_{\text {total }}=L_{\text {ant }}+L_{\text {match }}+L_{\text {air/ground }}+L_{\text {ground } / \text { air }}+L_{\text {spread }}+L_{\text {att }}+L_{\text {scat }}
$$


Similarly the received time domain waveform can be modelled as the convolution of several functions representing the impulse response of some component of the complete measurement environment (including noise sources etc). ${ }^{5}$

$$
f(t)_{\text {rec }}=f(t)_{\text {src }} * f(t)_{\text {ant }_{\text {tr }}} * f(t)_{\text {cross }} * f(t)_{\text {ground }} * f(t)_{\text {target }} * f(t)_{\text {ant }_{r c}} * f(t)_{\text {noise }},
$$

where,

$$
\begin{aligned}
& f(t)_{\text {src }}=\text { source signal presented to the transmitting antenna } \\
& f(t)_{\text {ant } t_{\text {tr }} \text { r }}=\text { impulse response of the transmit or receive antenna } \\
& f(t)_{\text {cross }}=\text { antenna cross coupling response } \\
& f(t)_{\text {ground }}=\text { impulse response of the ground } \\
& f(t)_{\text {target }}=\text { impulse response of the target } \\
& f(t)_{\text {noise }}=\text { noise }
\end{aligned}
$$

Equations 1 and 2 can be used to indicate where the environmental characteristics will impact upon the GPR signal. In this case we will investigate the influence of the soil water content on GPR measurements. Adding water to soil will affect the ground medium in several ways. For instance it may change the structure of the soil by rearranging the packing of the soil particles. Because the different electromagnetic characteristics of water, adding it to the soil, will effect in a change in electromagnetic characteristics somewhere in between those of the 'pure' soil and the 'pure' water. If the original soil contains a substantial amount of minerals these may be dissolved by the water introducing free ions in the medium thus raising the conductivity which will increase attenuation.

Table 1: Contributions to total path loss

\begin{aligned}$L_{\text {total }}= &$ Attenuation over the complete signal path \\ $L_{\text {ant }}= &$ Power available for radiation into the ground, as a proportion of the \\ & power applied to the antenna \\ $L_{\text {match }}= &$ Degree of mismatch between antenna and transmitter \\ $L_{\text {air } / \text { ground }}= &$ Transmission coupling loss. Represents the impedance mismatch \\ & between the air and soil medium \\ $L_{\text {ground } / \text { air }}= &$ Re-transmissions coupling loss. Represents the impedance mismatch \\ & during the return journey of the signal. (from the ground back up \\ & through the air into the receiving antenna) \\ $L_{\text {spread }}= &$ Antenna spreading loss is a number (conventionally) related to the \\ & inverse 4 th power of distance for a point scatterer (e.g. the radar- \\ & range equation). Non point-like scatterers like lines or planes will \\ & result in losses proportional to respectively the 3rd and 2nd inverse \\ & power of the distance \\ $L_{\text {att }}= &$ Depending on the nature of the (layered/inhomogeneous) embed- \\ & ding medium (soil) this can vary greatly. Moisture especially, has a \\ & big influence because it tends to free mineral ions that increase the \\ & conductivity of the ground and hence increase the loss. \\ $L_{\text {scat }}= &$ When the GPR signal reaches the target one gets the same kind of \\ & interface transition loss due to scattering. Sometimes the physical \\ & dimensions of the target and construction are such as to create a \\ & resonant structure which increases the level of the return signal and \\ & decreases the target scattering loss. \\ \hline & \end{aligned}

\subsection{Embedding soil properties}

Accurate modelling of the soil environment has been the subject of a lot of research and cannot be considered trivial due to the many degrees of freedom. Some of the models that are available give surprisingly good results 
if the soil or environmental parameters are not too extreme. However for gaining a basic understanding of the mechanisms involved and estimating the impact of change of soil characteristics on the GPR measurements a qualitative or empirical approach is sufficient.

The electromagnetic characteristics of materials are defined by their complex magnetic permeability $\mu$ and electric permittivity $\epsilon$. These are related to the wave speed in the material (soil medium) through the relation $v=1 / \sqrt{(} \mu \epsilon)$ (assuming that conductivity is very low). For free space (vacuum) these values are denoted as $\mu_{0}, \epsilon_{0}$ and light speed $c$ respectively. The relative permittivity which we will refer to from now on is defined through $\epsilon_{r}=c^{2} / v^{2}$ and $\epsilon=\epsilon_{0} \epsilon_{r}$. The relative permittivity lies in the range from 1 to approximately 80 for most geological materials, while the relative magnetic permeabillity can be taken as 1 for non magnetic geological materials, as is the case here.

The relative permittivity $\epsilon_{r}$ is strongly dependent on the volumetric water content $\theta_{v}$ of the soil. However it is almost independent of the soil density, texture and salt content at frequency between $20 \mathrm{MHz}$ and $1 \mathrm{GHz}$. In order to estimate the relative permittivity of the soil we will use the calibration curve reported by Topp et al. ${ }^{2}$ and use the (empirical) equation 3

$$
\epsilon_{r}=3.03+9.3 \theta_{v}+146 \theta_{v}^{2}-76.7 \theta_{v}^{3}
$$

For lossy dielectric materials attenuation of electromagnetic waves is due to both dielectric effects and conduction $\sigma$. One cannot through one single frequency measurement determine the separate components of loss for such materials. Table 2 shows some typical values of the conductivity and permittivity for air, water and some soil types.

Table 2: Typical range of dielectric characteristics of various materials at $100 \mathrm{MHz}$

\begin{tabular}{lcc}
\hline Material & Conductivity & Relative permittivity $\epsilon_{r}$ \\
\hline & $\mathrm{S} / \mathrm{m}$ & $\mathrm{F} / \mathrm{m}$ \\
Air & 0 & 1 \\
Water & $10^{-4}-10^{-2}$ & 81 \\
Clay dry & $10^{-3}-10^{-1}$ & $2-6$ \\
Clay saturated & $10^{-1}-1$ & $15-40$ \\
Concrete dry & $10^{-3}-10^{-2}$ & $4-10$ \\
Concrete wet & $10^{-2}-10^{-1}$ & $10-20$ \\
Sand dry & $10^{-7}-10^{-3}$ & $4-6$ \\
Sand saturated & $10^{-4}-10^{-2}$ & $10-30$ \\
Sandy dry soil & $10^{-4}-10^{-2}$ & $4-6$ \\
Sandy wet soil & $10^{-2}-10^{-1}$ & $15-30$ \\
Loamy dry soil & $10^{-4}-10^{-3}$ & $4-6$ \\
Loamy wet soil & $10^{-2}-10^{-1}$ & $10-20$ \\
Clayey dry soil & $10^{-4}-10^{-1}$ & $4-6$ \\
Clayey wet soil & $10^{-1}-1$ & $10-15$ \\
\hline
\end{tabular}

\section{EXPERIMENT}

To be able to test newly developed ultra wide band (UWB) GPR systems and to perform experiments in a controlled environment, a full scale experimental/test facility was erected on the premises of TNO-FEL. This facility has been developed in cooperation with the Delft University of Technology and is be used to illuminate objects buried in a realistic sand medium with an ultra-wide-band electromagnetic field. The electromagnetic field scattered by the buried object can be recorded and diagnosed for the benefit of developing a computer database containing the responses of a large number of potential targets (i.e. land mines etc.).

The experimental facility consists of a buried wooden box. The dimensions of this box are $10 \mathrm{~m} \times 10 \mathrm{~m}$ wide and $3 \mathrm{~m}$ deep. The box has been fully dug in into the ground, hence the bottom of the box is located three 
meters below ground level. Special care has been taken not to use any metal parts in the construction of the box or in the vicinity thereof. The sandbox is filled with relatively clean homogeneous river sand from the Dutch 'Calland' canal. In order to keep the condition of the sand in the box optimal and to prevent pollution from the outside (for example ground water) entering the box, a drainage system was installed and the inside of the box was covered with a watertight plastic lining. To prevent the weather from influencing the test conditions and to protect the measuring equipment, a large tent covers the entire site. Additionally, a wooden shed is available for storage of personal computers and measurement equipment. To facilitate the measurement of EM transmissions into the ground a square PVC tube running from the surface of one side to the bottom of the other side has been installed about one meter from the edge of the sandbox.

For the experiment described here, a makeshift test track was created about one meter form the edge of the sandbox. Three NR26 anti tank mines were buried at a depth of $30 \mathrm{~cm}$. Campbell CS615 water content reflectometers (WCR) were installed just above and just below the first mine at $29 \mathrm{~cm}$ and $43 \mathrm{~cm}$ respectively and at 5 and $15 \mathrm{~cm}$ below the surface. Two additional WCRs were installed in an empty area next to the AT mine at the same depth (see figure 2). The second AT mine was buried a little bit further on and is separated by an empty stretch of soil while the third AT mine is buried still further away in an area that is kept dry. We assume that the case of the anti tank mine will scale down to that of the anti personnel mine. The experiment however, was set up with anti tank mines because the WCR dimensions are of the same order than those of anti tank mines. The width of the test track was set at $40 \mathrm{~cm}$ and was dictated solely by the wheelbase of GPR 'trolley'. To support the wheels, wooden boards were placed on top of the loose sand on either side of the test track. The GPR used is a $1 \mathrm{GHz}$ SPR-scan system from ERA technology Ltd. UK. The choice for this system was purely based on practical considerations.

Before applying the water to the test area a couple of dry runs were made. Water equivalent to $80 \mathrm{~mm}$ rain was put in a number of big watering cans. It took two people approximately 5 minutes to distribute the water more or less evenly over the test track using these watering-cans. As soon as this was completed, GPR measurements commenced. Measurements were taken in the direction indicated in figure 2. Initially a GPR scan was made approximately every minute. After a while the frequency was dropped to a scan about every 5 minutes, 15 minutes and half hour. The next day and the following week two GPR scans per day were taken. The water content reflectometer (WCR) that were switched on beforehand were left running (logging) continuously.

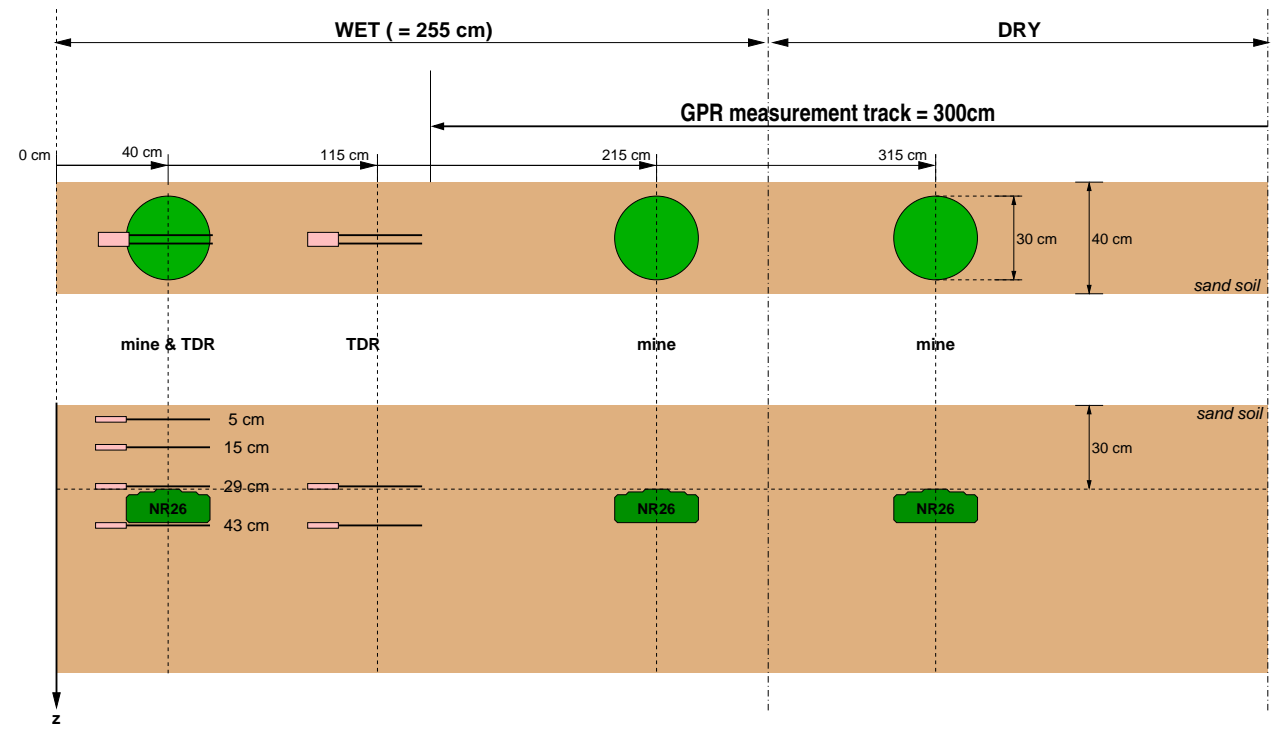

Figure 2: experimental setup of test track, a) plan view b) cross section 


\section{RESULTS}

\subsection{GPR measurements}

The GPR measurements result in images that show how a pulse is reflected and attenuated continuously through and by the soil medium. The images were created using the Seismic Un*x tool from the Colorado School of Mines, Center for Wave Phenomena. ${ }^{6}$ Figure 3a) shows the situation before any water was applied. Two boxes indicate where the NR26 anti-tank mines are located. Towards the end of the track one can already see the influence of the nearby WCR. Figure 3b) shows a GPR scan right after the water was applied. Most noticeable here is the huge reflection from the moisture front. Right behind the moisture front i.e. beyond the $200 \mathrm{~cm}$ mark, the propagation speed of the electromagnetic waves in the soil medium is reduced considerably. The two-way travel times are longer and the features in the picture are elongated in the time. Apart from that the water in the soil attenuates the signal making it harder to detect beyond the moisture front. In figure 4 the

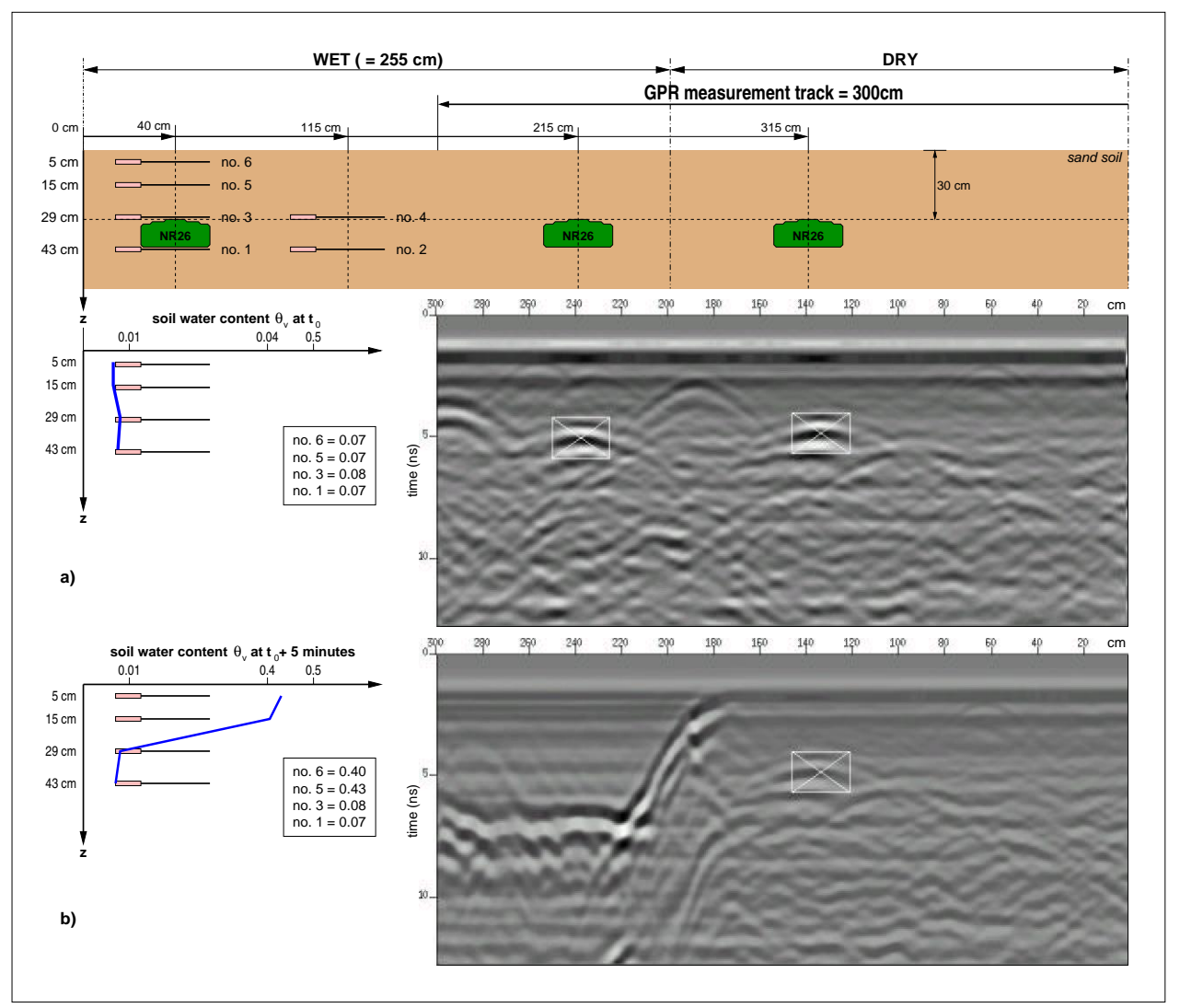

Figure 3: results of GRP measurements a) 'dry' initial situation b) 'wet' situation directly after introducing water

situation after 5 and after 51 hours is shown. The wave speed is still substantially lower than in the initial (dry) state. Figure 4 a) (after 5 hours) shows an edge effect around the AT-mine. This is due to the sudden change in moisture levels and thus also soil permittivity. In figure $4 \mathrm{~b}$ ) (after 51 hours) the wave speed has returned almost to the initial value. Unfortunately the location of the AT-mine was chosen a bit too close to the border between the dry and wet area. This causes the reflection of the moisture front to overshadow the reflection of the AT-mine, making it difficult to discern the hyperbola. Nevertheless one can see a much stronger reflection there than in the dry part.

\subsection{Measurements of soil water content}

Figure 5 shows the soil water content recordings near the AT-mine over a period of 50 hours after infiltration on 1:00 pm February 16, 2002. Between 1:00 pm and 1:05 pm $80 \mathrm{~mm}$ of water was applied (Note that this is 


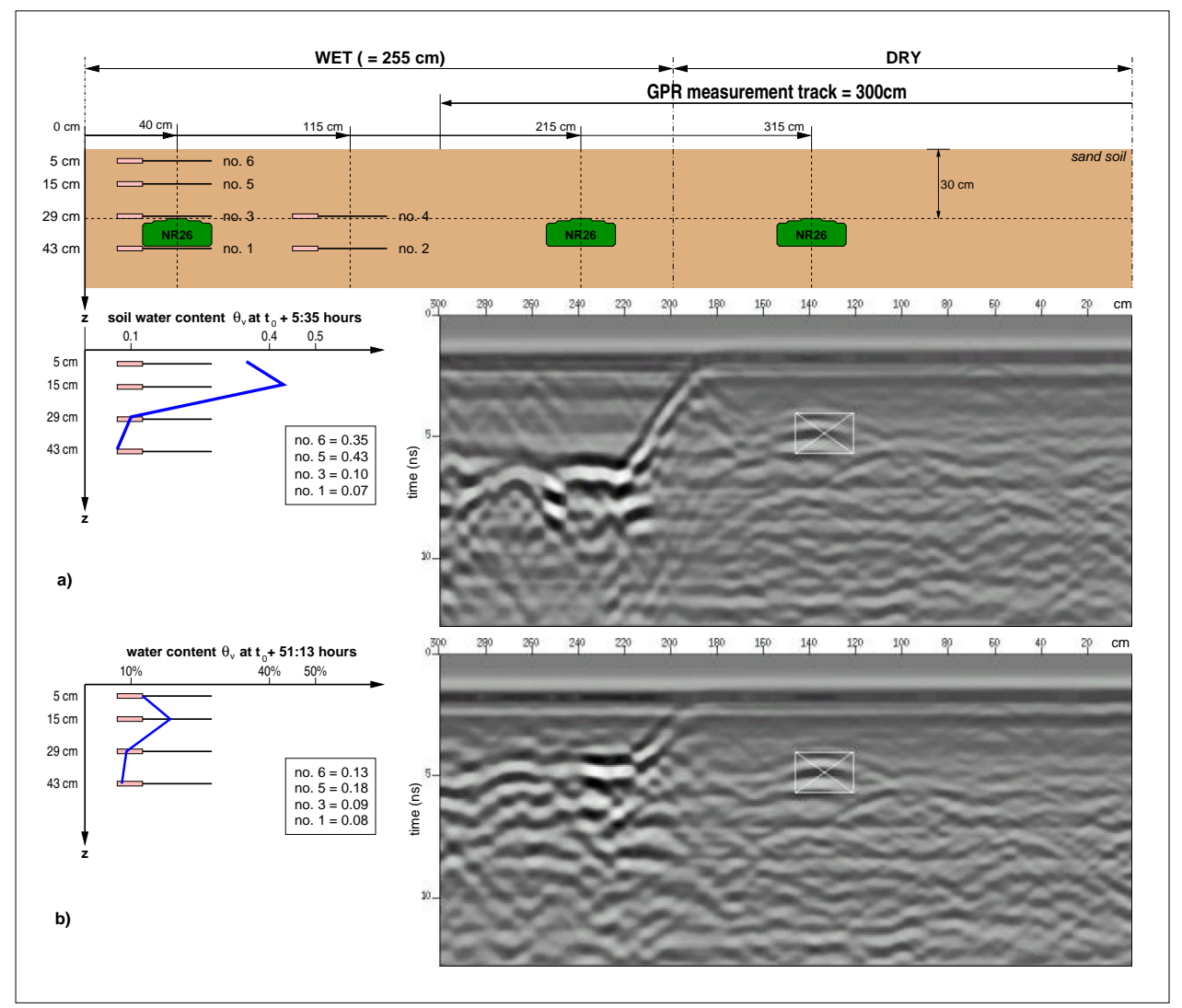

Figure 4. results of GPR measurements a) 5:35h after the start of the experiment b) 51:13h after start of the experiment

Table 3: Soil water content $\theta_{v}\left[\mathrm{~m}^{3} / \mathrm{m}^{3}\right]$ as a function of depth and time after infiltration

\begin{tabular}{cccccc}
\hline WCR & Depth & $\theta_{v}\left(t_{0}\right)$ & $\theta_{v}\left(t_{0}+0 \mathbf{h} 05\right)$ & $\theta_{v}\left(t_{0}+5 \mathbf{h} 35\right)$ & $\theta_{v}\left(t_{0}+51 \mathbf{h} 13\right)$ \\
\hline no.1 & $43 \mathrm{~cm}$ & 0.07 & 0.07 & 0.07 & 0.08 \\
no. 2 & $43 \mathrm{~cm}$ & 0.06 & 0.06 & 0.06 & 0.06 \\
no.3 & $29 \mathrm{~cm}$ & 0.08 & 0.08 & 0.10 & 0.09 \\
no. 4 & $29 \mathrm{~cm}$ & 0.07 & 0.07 & 0.13 & 0.11 \\
no.5 & $15 \mathrm{~cm}$ & 0.07 & 0.43 & 0.43 & 0.18 \\
no. 6 & $5 \mathrm{~cm}$ & 0.07 & 0.40 & 0.35 & 0.13 \\
\hline
\end{tabular}


an extreme high intensity ca. $960 \mathrm{~mm} / \mathrm{h}$ !). From the start and during of the experiment the water content was measured continuously at $5 \mathrm{~cm}, 15 \mathrm{~cm}, 29 \mathrm{~cm}$ and $43 \mathrm{~cm}$ depth. The recordings at $29 \mathrm{~cm}$ and $43 \mathrm{~cm}$ depth were done just above and just below the AT-mine, and at horizontal distance of $35 \mathrm{~cm}$ (reference area). From the

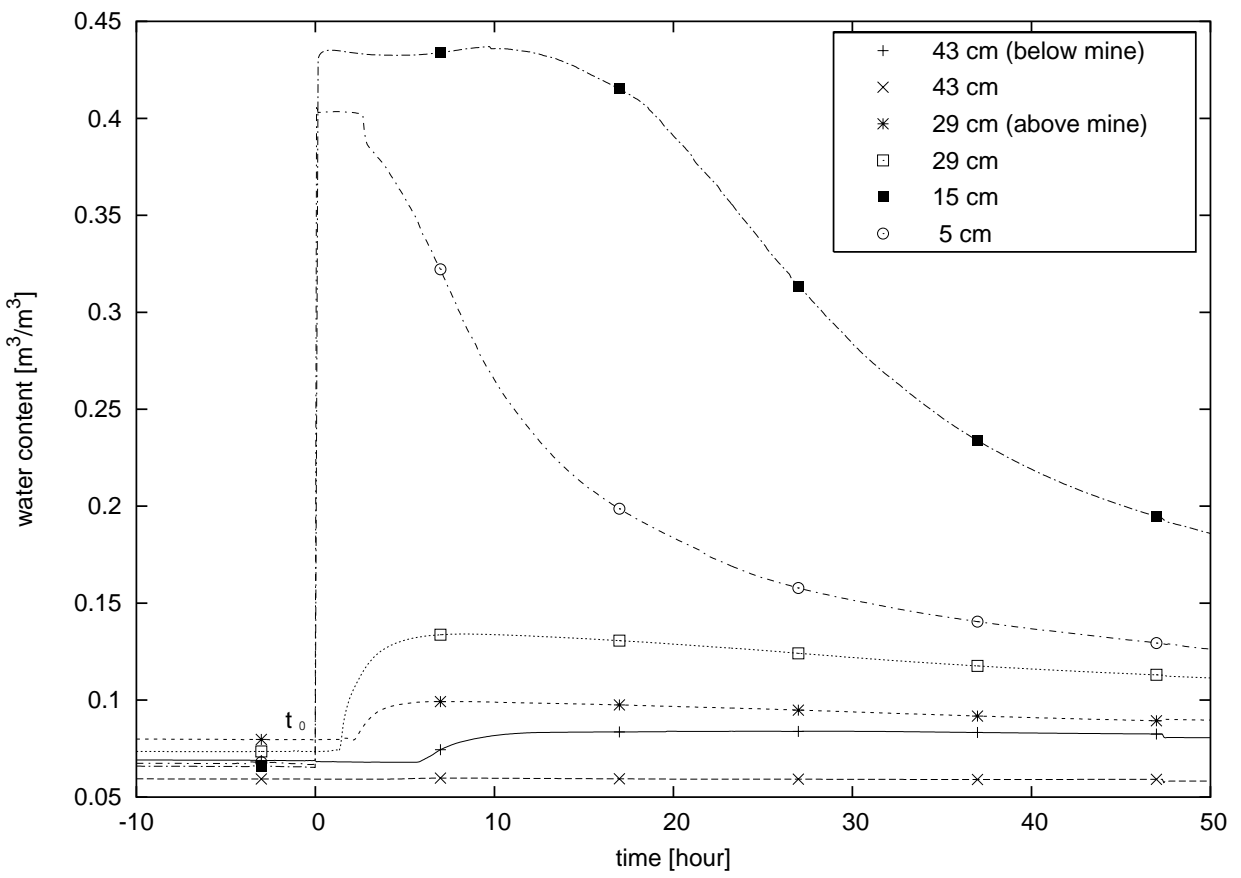

Figure 5: Measured water content after $80 \mathrm{~mm}$ infiltration

figure some features are noted. Except for a depth of $43 \mathrm{~cm}$ in the reference area, the soil water content increased after infiltration and after some while decreases and stabilises around some average value. The reaction time is generally short: several minutes, $1.4 \mathrm{hrs}, 2.2 \mathrm{hrs}$ and $6.8 \mathrm{hrs}$, respectively for $5 \mathrm{~cm}$ and $15 \mathrm{~cm}, 29 \mathrm{~cm}$ and $43 \mathrm{~cm}$ depth. The sensor in the reference area at depth $43 \mathrm{~cm}$ did not respond (no increase of water content). The peaks in the curves are more spread out with increased depth.

After infiltrating $80 \mathrm{~mm}$ water the water content above the AT-mine increased from initially 0.08 to $0.10\left[\mathrm{~m}^{3} / \mathrm{m}^{3}\right]$ and below the mine from 0.07 to $0.08\left[\mathrm{~m}^{3} / \mathrm{m}^{3}\right]$. The maximum water content of $0.44\left[\mathrm{~m}^{3} / \mathrm{m}^{3}\right]$ was measured at the depth of $15 \mathrm{~cm}$.

A few things can be noted when comparing the measured with the modelled water content values (see also figure 1). The modelled maximum water content values are lower than the measured values. This may be due to the lower simulated precipitation intensity $(80 \mathrm{~mm} / \mathrm{h})$ and an assumed higher hydraulic conductivity of the model. The latter probably also is responsible for the steeper depletion curve. Further, the water content below the mine increases earlier during the experiment than calculated with the model. This is maybe the result of leakage of water around the mine. Due to the burial activities the structure of the sand around the mine is probably relative open compared to the surrounding area. Compare the values of the area at a depth of $43 \mathrm{~cm}$ near the mine, which remained the same $\left(0.06\left[\mathrm{~m}^{3} / \mathrm{m}^{3}\right]\right)$ during the experiment. Note that during the experiment water could be lost as a result of lateral flow. This would explain the lower values measured at $29 \mathrm{~cm}$ and $43 \mathrm{~cm}$ depth and the convergence of all the measured values around $0.1\left[\mathrm{~m}^{3} / \mathrm{m}^{3}\right]$ after $250 \mathrm{~h}$. The modelled values seem to stabilise around $0.15\left[\mathrm{~m}^{3} / \mathrm{m}^{3}\right]$ after $50 \mathrm{~h}$.

\section{ANALYSIS}

There are several ways in which one can try to determine the bulk permittivity or permittivity profile of the embedding. Probably the most simple way to determine the effective bulk permittivity of the soil between the 
GPR antenna and the land mine target is to use the two way travel time between the antenna and target together with the known depth of the target. Normally one does of course not have this a priori knowledge and one is forced to use a bit more elaborate measurements e.g. common depth point (CDP) measurements together velocity analysis schemes, hyperbolic spreading function, or more advanced calculations like electromagnetic inverse profiling schemes. We will for the time being, revert to the more basic determination of wave speed through the use of the two way travel time $t_{r}$ and the known depth of the target $d$ shown in equation 4

$$
v=2 d / t_{r}
$$

Equation 4 however does not take the antenna separation into account. It assumes that the location of transmit and receive antenna coincide. A lot of commercial pulsed ground penetrating radar systems however work in common offset mode. In the case of the ERA SPR-scan system with the $1 \mathrm{GHz}$ antenna the distance between the transmit and receive antennas (offset) is $111 \mathrm{~mm}$. This affects the calculation of wave speed for shallow buried (reference) targets. To correct for this and to take into account the location of the antenna above the ground (in our case $\approx 1 \mathrm{~cm}$ ) we introduce the modified form of equation 4

$$
v=2 \frac{\sqrt{d^{2}+a^{2}}}{t_{r}-2 t_{\text {air }}}
$$

Where $a=\frac{1}{2}$ of the antenna separation and $t_{\text {air }}=$ part of the travel time through air before the wave enters the ground $(1 \mathrm{~cm} \approx 33.4 \mathrm{ps})$.

Measuring the time of the first reflections from the data in figure $3 \mathrm{a}$ ) and $3 \mathrm{~b}$ ) we get $3.9 \mathrm{~ns}$ and $6.3 \mathrm{~ns}$ respectively. Putting this in equation 5 yields wave speed values of $v=1.59 \cdot 10^{8} \mathrm{~m} / \mathrm{s}$ and $v=0.98 \cdot 10^{8} \mathrm{~m} / \mathrm{s}$ and corresponding values of the (bulk) relative permittivity $\epsilon_{r}=3.5$ and $\epsilon_{r}=9.4$ (keeping in mind that $v=c / \sqrt{\epsilon_{r}}$ ). As one can also see from figure 3 the contrast of the anti tank mine with its surrounding soil environment is not very high. Although visible the strength of the return is not all that strong. According to the Dow Corning product information ${ }^{7}$ the relative permittivity $\epsilon_{r}$ of the RTV compound used in the anti tank mine simulant is 2.9 at $1 \mathrm{MHz}$ and 2.89 at $1 \mathrm{GHz}$. This is pretty close to the calculated bulk permittivity of the soil environment. Watering the test area raises the bulk relative permittivity substantially and thus created a bigger contrast. However, from figure $4 \mathrm{a}$ ) and figure $4 \mathrm{~b}$ ) it is clear that the reason the land mine sticks out is not just through the change in relative permittivity of its surrounding but also and maybe especially from figure 4a) because water collects at the top of the mine and then flows around then mine (i.e. a very local effect). This is also supported when looking at figure $4 \mathrm{~b}$ ) and realising that the bulk wave speed must be almost back to normal when looking at the two way travel times. Yet the land mine is much better visible due to the wetness of the land mine surface (i.e. water that collected on top). So it would maybe be better to say that the introduction of moisture/water in the environment creates a so called water or moisture 'shadow' around the land mine which makes it far more noticeable than the change in contrast due to the overall change in medium permittivity.

When inserting the soil water content values $\theta_{v}$ measured in the experiment into equation 3 we get the results like depicted in table 4 . The values we determined earlier on by calculating the wave speed from the

Table 4: Soil water content $\theta_{v}\left[\mathrm{~m}^{3} / \mathrm{m}^{3}\right]$ and relative permittivity $\epsilon_{r}$ as a result from Topp's equation

\begin{tabular}{ccccccccccc}
\hline \multirow{2}{*}{ WCR } & \multirow{2}{*}{ Depth } & \multicolumn{3}{c}{$t_{0}$} & \multicolumn{2}{c}{$t_{0}+0 \mathrm{~h} 05$} & \multicolumn{2}{c}{$t_{0}+5 \mathrm{~h} 35$} & \multicolumn{2}{c}{$t_{0}+51 \mathrm{~h} 13$} \\
\cline { 3 - 11 } & & $\theta_{v}$ & $\epsilon_{r}$ & $\theta_{v}$ & $\epsilon_{r}$ & $\theta_{v}$ & $\epsilon_{r}$ & $\theta_{v}$ & $\epsilon_{r}$ \\
\hline no.1 & $43 \mathrm{~cm}$ & 0.07 & 4.4 & 0.07 & 4.4 & 0.07 & 4.4 & 0.08 & 4.7 \\
no.2 & $43 \mathrm{~cm}$ & 0.06 & 4.1 & 0.06 & 4.1 & 0.06 & 4.1 & 0.06 & 4.1 \\
no.3 & $29 \mathrm{~cm}$ & 0.08 & 4.7 & 0.08 & 4.7 & 0.10 & 5.3 & 0.09 & 5 \\
no.4 & $29 \mathrm{~cm}$ & 0.07 & 4.4 & 0.07 & 4.4 & 0.13 & 6.5 & 0.11 & 5.7 \\
no.5 & $15 \mathrm{~cm}$ & 0.07 & 4.4 & 0.43 & 28 & 0.43 & 28 & 0.18 & 9 \\
no.6 & $5 \mathrm{~cm}$ & 0.07 & 4.4 & 0.40 & 25 & 0.35 & 25 & 0.13 & 6.5 \\
\hline \multicolumn{2}{l}{ effective avg. $\epsilon_{r}$} & & 4.5 & & 19.7 & & 20 & & 7.1 \\
\hline
\end{tabular}

two way travel time, represent the average bulk relative permittivity of the soil between the antenna and the 
mine. If we want to compare the numbers in table 4 derived from Topp's model through equation 3 we will have to do this through calculation of some kind of average from them. To this end we observe that a plane wave travelling in the direction of the $z$-axis through a medium with wave speed $v$ is written as,

$$
\exp [-j \omega z / v]
$$

For a 'continuously layered' medium assuming only waves travelling in the positive $z$-direction this expression becomes,

$$
\exp \left[-j \omega \int \frac{1}{v(z)} \mathrm{d} z\right] .
$$

Using the fact that $v(z)=c / \sqrt{\mu_{r}(z) \epsilon_{r}(z)}$ and assuming that $\mu_{r}(z)=1$ we obtain for the 'layer' $0<z<d$,

$$
\exp \left[-j \frac{\omega}{c} \int_{0}^{d} \sqrt{\epsilon_{r}(z)} \mathrm{d} z\right] .
$$

This implies that, if we are able to determine the permittivity of some medium at several depths (locations) and want to compare these values with the permittivity found by using the simple method of equation 5 , we should approximate the integral $\int_{0}^{d} \sqrt{\epsilon_{r}(z)} \mathrm{d} z$ by means of something like the trapezoïdal rule. Because, if $\epsilon_{r}$ were to be constant we would get,

$$
\int_{0}^{d} \sqrt{\epsilon_{r}(z)} \mathrm{d} z=d \sqrt{\epsilon_{r e}}
$$

where $d$ is the known depth of the object. This means that $\epsilon_{r e}$ ('re' for relative effective) represents the value that we would get from equation 5 and the fact that $v=c / \sqrt{\epsilon_{r}}$. When calculating $\epsilon_{e}$ we assumed that the value for $\epsilon_{r}$ at the surface is the same as at a depth of $5 \mathrm{~cm}$. To approximate the integral, the trapezoïdal rule was used. The outcome is listed at the bottom of table 4 . These values deviate quite a bit from the values we got earlier on in this section. The trend however is correct. Nevertheless one would want an accuracy in the same order at the expected impedance (dielectric) contrast. Ideally one would implement an inverse profiling scheme designed to reconstruct the permittivity profile of the soil environment. This however, is far from straight forward and beyond the scope of this paper.

\section{CONCLUSIONS}

When comparing the HYDRUS-2D model with the measurement results we see a close resemblance between the modelled moisture distribution and the actual measurements. Reasons for deviations have been discussed previously in the paper.

Although the absolute values of the measured and modelled water content are different, the shapes of the curves are similar. Better model predictions are expected when the hydraulic properties of the soil surrounding the mine are more accurately know.

We see, not surprisingly, that introducing water to a (dry) sand soil environment greatly changes the response of the GPR. Right after applying the water we see a moisture front that significantly attenuates the signal thereby blocking out deeper located features. There are several instances in time during the infiltration process that are interesting with respect to mine detectability. The first interesting instance is at $t_{0}+5$ hours. 'Leakage' around the edges of the mine is quite prominent. This can maybe be used to make a rough estimate of the dimension(s) of the mine. The second interesting moment is at about $t_{0}+5$ hours. Here we see that the surrounding soil environment has substantially dried out and is returning towards the initial state. The impedance contrast of the land mine with respect to its surrounding (i.e. stronger electromagnetic reflections) has increased which can result in an enhanced 'visibility' of buried objects like land mines. Alternative effects also seem to play a role in finding and identifying features of potential targets. We see a strong reflection from the soil/mine interface at time $t_{0}+51 \mathrm{~h} 13$ and suspect this is due to a film of water on the surface of the mine (i.e. highly localised effect). 


\section{ACKNOWLEDGMENTS}

This work is funded by a grant from the Army Research Office (Project 38830-EL-LMD). The authors would like to thank Dr. Russell S. Harmon, Senior Program Manager at the Army Research Office, for his valuable advice and support. In addition the authors wish to thank Mr. P.J. Fritz for his technical assistance during the measurements.

\section{REFERENCES}

1. J. Šimůnek, M. Šejna, and M. T. van Genuchten, HYDRUS-2D/MESHGEN-2D code for simulating water flow and solute transport in two-dimensional variably saturated media. International Ground Water Modeling Center, Colorado School of Mines, CO 80401, USA., gwmc - tps 53c, ver. 2.0 ed.

2. G. C. Topp, J. L. David, and A. P. Annan, "Electromagnetic determination of soil water content: Measurements in coaxial transmission lines," Water Resources Research 16, pp. 574-582, June 1980.

3. B. S. Das, J. M. H. Hendrickx, and B. Borchers, "Modeling transient water distributions around landmines in bare soils.," Soil Science 166, 2001. in press.

4. J. M. H. Hendrickx, B. S. Das, and B. Borchers, "Modeling distributions of water and dielectric constants around landmines in homogeneous soils.," in Detection and Remediation Technologies for Mines and Minelike Targets IV, Proceedings of the SPIE 3710, pp. 728-738, 1999.

5. D. J. Daniels, Surface Penetrating Radar, The Institute of Electrical Engineer (IEE), 1996.

6. J. K. Cohen and J. W. Stockwell Jr., CWP/SU: Seismic Unix Release 35: a free package for seismic research and processing. Center for Wave Phenomena, Colorado School of Mines, CO 80401, USA., 2001.

7. Dow Corning Europe, Brussels, Product Information for Silastic 3110 RTV Silicone Rubber, 1981. Data Sheet number 61-143-01. 